\title{
Project Startup: Evaluating the Performance of Frito Lay's Electric Delivery Trucks
}

The Fleet Test and Evaluation Team at the National Renewable Energy Laboratory (NREL) is evaluating the in-service performance of 10 medium-duty Smith Newton electric vehicles (EVs) and 10 comparable conventional diesel vehicles operated by Frito Lay North America in the Seattle, Washington, area. This in-depth study complements a broader, nationwide evaluation (described at the end of this section) of more than 600 EVs deployed by a variety of companies.

Launched in late 2013, the on-road portion of this 12-month evaluation focuses on collecting and analyzing vehicle performance data, such as fuel economy and maintenance costs, to better understand how to optimize the use of such vehicles in a large-scale commercial operation.

EVs can reduce fuel costs dramatically because of the low cost of electricity relative to conventional fuel. The fuel economy of medium- and heavy-duty EVs is largely dependent on the load carried and the drive cycle. In the right applications, EVs can maintain a strong fuel-cost advantage over their conventional counterparts. This advantage is contingent, however, on charging during the appropriate times of day (i.e., when utility rates are lower). NREL's evaluation will help Frito Lay determine the delivery routes and charging schedules that maximize the fuel-saving potential of its EVs.

In addition to the on-road portion of this evaluation, NREL is analyzing charging data to support total cost of ownership estimations and investigations into smart charging opportunities. Smart charging takes into consideration a variety of factors - off-peak timing, driving range requirements, and other facility or utility needs - to minimize the direct and indirect costs of vehicle charging.

NREL is also performing a battery life degradation analysis to quantify battery pack health, track battery performance over time, and determine how various drive

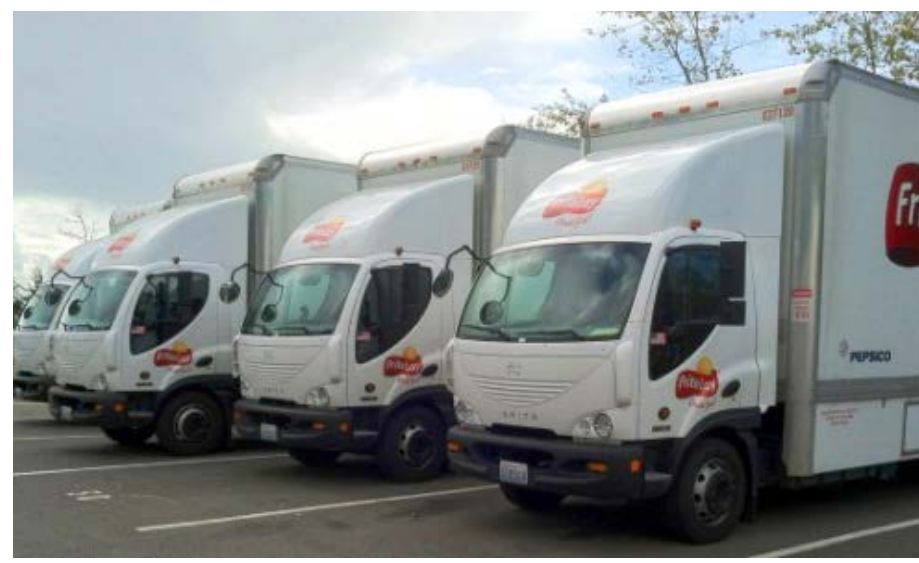

Frito Lay operates Smith Newton electric vehicles out of its fleet depot in Seattle. Photo by Mike Simpson, NREL 28804

cycles and battery charging protocols impact battery life. NREL will use the measured rate of battery pack degradation along with a battery life prognostic model to forecast the life of the battery and the associated operational costs.

This multi-pronged evaluation builds on a larger NREL evaluation of more than 600 medium-duty EVs deployed by a variety of companies, including Frito Lay, across the nation with support from the American Recovery and Reinvestment Act. The larger study aims to improve understanding of the overall usage and effectiveness of electric fleet vehicles compared to similar conventional vehicles operating in the same area.

\section{Frito Lay's Electric Delivery Vehicles}

Frito Lay operates more than 250 electric delivery vehicles as part of its commitment to environmental sustainability. With zero tailpipe emissions and a top speed of $55 \mathrm{mph}$, the Smith Newton EV has a range of 40-100 miles on a single charge. It features a brushless, permanent magnet motor with134-kW peak power and lithium-ion batteries that provide about 350 VDC and 80 or $120 \mathrm{kWh}$ of energy storage, depending on the 
configuration. Some energy that is normally lost during braking is captured, stored in the batteries, and used to power the electric motor. Because of these regenerative braking capabilities, the vehicle can operate at peak effectiveness in urban applications with heavy stop-andgo driving.

\section{In-Service Testing in the Seattle Region}

NREL started its on-road evaluation of the 10 conventional vehicles in November 2013, while data collection from the 10 EVs started in January 2013. The vehicles serve similar routes in Frito Lay's Seattle delivery area. The study vehicles may switch routes mid-way through the evaluation to improve the comparability of the routes driven by each vehicle type over the entire study period.

NREL will use the following resources to compare the fuel economy, maintenance, and durability of the electric and conventional vehicles:

- Vehicle fueling/charging data from fueling/charging records, engine control modules, and data loggers

- Vehicle mileage data from driver logs, engine control modules, and data loggers

- Vehicle maintenance data from service reports.

\begin{tabular}{|l|l|l|}
\hline & Electric & Conventional Diesel \\
\hline Chassis manufacturer/model & Smith Electric Vehicles/Smith Newton & International/Hino \\
\hline $\begin{array}{l}\text { Chassis model year } \\
\text { Engine ratings }\end{array}$ & 2013 & $2001-2013$ \\
\hline $\begin{array}{l}\text { Maximum horsepower } \\
\text { Maximum torque }\end{array}$ & $180 \mathrm{HP}$ & \\
\hline Curb weight & $480 \mathrm{lb}-\mathrm{ft}$ & $175-325 \mathrm{HP}$ \\
\hline Regenerative braking & $\sim 9,500 \mathrm{lbs}$ & $376-570 \mathrm{lb}-\mathrm{ft}$ \\
\hline Advanced emission-control equipment & Yes & $\sim 8,000 \mathrm{lbs}$ \\
\hline
\end{tabular}

\section{Advanced Vehicle Testing}

This project is part of a series of evaluations performed by NREL's Fleet Test and Evaluation Team for the U.S. Department of Energy's Advanced Vehicle Testing Activity (AVTA) with funding from the Vehicle Technologies Office. AVTA bridges the gap between R\&D and the commercial availability of advanced vehicle technologies that reduce petroleum use and improve air quality in the United States. AVTA projects provide comprehensive, unbiased evaluations of advanced vehicle technologies in commercial use. Data are collected and analyzed for operation, maintenance, performance, cost, and emission characteristics of advanced technology fleets and comparable conventional technology fleets operating at the same site. These evaluations enable fleet owners and operators to make informed vehicle-purchasing decisions.

\section{More Information}

For more information about this and other evaluations conducted by NREL's Fleet Test and Evaluation Team, refer to the website at www. nrel.gov/vehiclesandfuels/ fleettest. 